\title{
BRMS1 expression in resected lung adenocarcinoma
}

\author{
Domenico Galetta, Pamela Pizzutilo, Vito Longo \\ Medical Thoracic Oncology Unit, IRCCS Istituto Tumori “Giovanni Paolo II”, Bari, Italy \\ Correspondence to: Vito Longo, MD, PhD. Medical Thoracic Oncology Unit, IRCCS Istituto Tumori “Giovanni Paolo II”, Viale Orazio Flacco 65 - \\ 70124, Bari, Italy. Email: v.longo@oncologico.bari.it. \\ Comment on: Bucciarelli PR, Tan KS, Chudgar NP, et al. BRMS1 Expression in Surgically Resected Lung Adenocarcinoma Predicts Future \\ Metastases and Is Associated with a Poor Prognosis. J Thorac Oncol 2018;13:73-84.
}

Submitted Sep 16, 2018. Accepted for publication Sep 25, 2018.

doi: 10.21037/tlcr.2018.09.20

View this article at: http://dx.doi.org/10.21037/tlcr.2018.09.20

The identification of prognostic markers for surgical resected cancers is a current issue for several malignancies $(1,2)$. As regard lung adenocarcinoma (LUAD), despite curative-intent surgical resection, tumor recurrence and spread remain the primary causes of cancer-related death among patients with early stage. A precise prediction of the risk of tumor recurrence at the time of surgery could spare patients the toxicity of adjuvant chemotherapy, and target other patients for increased therapy and surveillance (3). Differently from advanced stages, risk factors to identify patient with surgically resectable LUAD who have high risk of recurrence are poorly defined. TNM stage, tumor size, lympho-vascular invasion, and visceral pleural invasion are the main prognostic factors for resected LUAD (4). Moreover, others risk factors are now under investigation, such as architectural grade, carcinogenembryonic antigen (CEA) levels, standardized uptake value (SUV) of positron emission tomography (PET), thyroid transcription factor (TTF)-1 expression levels, mutations in KRAS gene, tumor protein 553 gene, phosphatidylinositol-4,5-bisphosphate 3 -kinase catalytic subunit alpha, single nucleotide polymorphisms, and microRNA (4-8).

Selected metastasis suppressor genes are also other potential prognostic factors for resect LUAD. For example, decreased levels of CD82 molecule gene (KAI1) and KISS1 metastasis-suppressor gene (KISS1) have been associated with poor prognosis in a small series of lung cancers $(9,10)$. Among selected metastasis suppressor genes, the breast cancer metastasis suppressor 1 (BRMS1) gene is one of the most promising as prognostic factors in LUAD. BRMS1 maps to chromosome 11q13.1-13.2 and contains a helix-turn-helix DNA binding domain and coiled-coiled domains, suggesting that it may be part of a transcription complex (11). BRMS1 has been shown to function as a corepressor to inhibit NF$\mathrm{kB}$ transactivation by deacetylation of the RelA/p65 subunit at K310. It also regulates angiogenesis, phosphoinositide signalling, expression of microRNA, and p300 histone acetyltransferase levels. Moreover, the loss of endogenous BRMS1 significantly promotes basal and TGF-Beta-induced epithelial to mesenchymal transition (EMT) in lung cancer cells (12).

A reduced BRMS1 expression has been identified as a predictive factor of poor prognosis in breast cancer (13), nasopharyngeal carcinoma (14), gallbladder adenocarcinoma (15), and melanoma (16).

Smith and co-workers first reported that BRMS1 expression was reduced in NSCLC cells and human tumor tissue correlating with worse overall survival (OS) (17). Loss of BRMS1 expression is related to promoter methylation by RelA/p65-DNA methyltransferase and to phosphorylation on serine 30 by CK2 $\alpha$ resulting in 14-3-3 mediated nuclear exportation of BRMS1 and following proteasome-mediated ubiquitination and degradation. Interestingly, methylation of BRSM1 promoter correlate with smoking history and poor survival (18).

More recently, the same research group has been investigated the BRMS1 expression in 1,030 patients who underwent complete resection for LUAD (19). This large cohort, included patients from stage I to stage IIIA with a prevalence of stage I patients (85\%), median followup was 5-years. BRMS1 expression was evaluated on the basis of intensity of nuclear immunostaining, with $0+$ indicating no staging and $1+, 2+, 3+$ indicating weak, moderate, and strong staging respectively. The low 
BRMS1 expression was defined as an intensity score of 0 to 2 and the high expression as a score of 3. Low BRMS1 expression was associated with grater tumor size, higher pathologic stage, and greater lymphatic and vascular invasion. Furthermore, BRMS1 expression had also a strong correlation with LUAD histologic subtype and architectural grade. In particular, micropapillary and solid predominant LUAD had low BRMS1 expression, on the other hand lepidic predominant subtype were more common in the BRMS1 high-expression group. In accord with the determinations in previous small series, Bucciarelli et al. confirmed that low BRMS1 expression was associated to poor prognosis, resulting as an independent predictor of worse OS [hazard ratio (HR) $=1.35,95 \%$ confidence interval: $1.10-1.65, \mathrm{P}=0.004]$ and disease-free survival (DFS) ( $\mathrm{HR}=1.27,95 \%$ confidence interval: $1.05-1.54, \mathrm{P}=0.012)$.

Despite the study elegantly examined the role of BRMS1 in a very large cohort of resected LUAD, the contribution of this single biomarker in a multivariate model including standard clinicopathologic features as age, sex, surgery type, pathologic stage, tumor size, lymphovascular invasion, and histologic subtype seems small. Even if, the study cohort is characterized for $85 \%$ by stage I patients, the presence of patients with pathological stages other than I, in particular about $7 \%$ of stage IIIA, could be mystifying. As regards, a retrospective subgroup analysis, evaluating the prognostic role of BRMS1 levels for different stage could be performed. Interesting, in addition to BRMS1 immunohistochemical analysis, Bucciarelli and co-workers showed in two independent cohorts of stage I LUAD without nodal metastasis, namely Nagoya cohort $(\mathrm{n}=79)$ and University of Michigan cohort $(\mathrm{n}=128)$, that low BRMS1 mRNA transcript levels significantly correlated with decreased OS. According to this, immunohistochemical and mRNA BRMS1 expression could be prospectively evaluated focusing pathologic stages that are borderline for adjuvant chemotherapy such as T2a-b without nodal metastasis, evaluating thus a potential predictive role of this biomarker.

Analogous to the paper of Bucciarelli and colleagues, in the last ten years, several studies identified immunohistochemical expression of single protein as potential prognostic factor for resected lung cancer, such as AAA+ nuclear coregulator cancer associated (ANCCA) (20), phosphatase and tensin homolog (PTEN) (21), baculoviral inhibitors of apoptosis proteins repeat-containing 6 (BIRC6) protein (22), transcriptional coactivator with PDZ-binding motif (TAZ) (23), mammalian target of rapamycin (mTOR) (24), disintegrin (25), metalloproteinase-9 (ADAM9) (25), and others. Unfortunately, nowadays none of these potential prognostic factors is entered in clinical practice, both difficulties to reproducibility and implementation, as well as a low representativeness of all pathways implicated in tumor progression by a single biomarker, have prevented the widespread use of these biomarkers in the clinic.

Probably, the combinatory analysis of BRMS1 expression with the expression levels of other factors implicated in tumor growth, angiogenesis, and tumor progression could results in a more effective prognostic tool. As it happens for other malignancies, for example about hormone positive resected breast cancer with the Food and Drug Administration (FDA) approved gene expression profiling, namely Oncotype DX and MammaPrint (1) or about gastric cancer with tool under clinical investigation, as the use of The Cancer Genome Atlas (TCGA) project and the Asian Cancer Research Group (ACRG) molecular classifications (2).

Be that as it may, Bucciarelli and co-workers, are to be congratulated on their contribution about the study of resected LUAD prognosis, using a very large cohort of resect LUAD with predominant stage I patients. They have identified a new potential biomarker that can be validated in new under development approaches combining both transcriptomic and proteomic data related to tumor progression pathways.

\section{Acknowledgement}

None.

\section{Footnote}

Conflicts of Interest: The authors have no conflicts of interest to declare.

\section{References}

1. Bhutiani N, Egger ME, Ajkay N, et al. Multigene Signature Panels and Breast Cancer Therapy: Patterns of Use and Impact on Clinical Decision Making. J Am Coll Surg 2018;226:406-12.e1.

2. Battaglin F, Naseem M, Puccini A, et al. Molecular biomarkers in gastro-esophageal cancer: recent developments, current trends and future directions. Cancer Cell Int 2018;18:99.

3. Martini N, Bains MS, Burt ME, et al. Incidence of local recurrence and second primary tumors in resected stage I lung cancer. J Thorac Cardiovasc Surg 1995;109:120-9. 
4. Koo HK, Jin SM, Lee CH, et al. Factors associated with recurrence in patients with curatively resected stage I-II lung cancer. Lung Cancer 2011;73:222-9.

5. Kadota K, Nitadori J, Sarkaria IS, et al. Thyroid transcription factor-1 expression is an independent predictor of recurrence and correlates with the IASLC/ ATS/ERS histologic classification in patients with stage I lung adenocarcinoma. Cancer 2013;119:931-8.

6. Nadal E, Chen G, Prensner JR, et al. KRAS-G12C mutation is associated with poor outcome in surgically resected lung adenocarcinoma. J Thorac Oncol 2014;9:1513-22.

7. Zhang L, Shi L, Zhao X, et al. PIK3CA gene mutation associated with poor prognosis of lung adenocarcinoma. Onco Targets Ther 2013;6:497-502.

8. Hong MJ, Choi YY, Jang JA, et al. Association between genetic variants in pre-microRNAs and survival of earlystage NSCLC. J Thorac Oncol 2013;8:703-10.

9. Sun YB, Xu S. Expression of KISS1 and KISS1R (GPR54) may be used as favorable prognostic markers for patients with non-small cell lung cancer. Int J Oncol 2013;43:521-30.

10. Zhou L, Yu L, Wu S, et al. Clinicopathological significance of KAI1 expression and epithelial-mesenchymal transition in non-small cell lung cancer. World J Surg Oncol 2015;13:234.

11. Seraj MJ, Samant RS, Verderame MF, et al. Functional evidence for a novel human breast carcinoma metastasis suppressor, BRMS1, encoded at chromosome 11q13. Cancer Res 2000;60:2764-9.

12. Liu Y, Mayo MW, Xiao A, et al. Loss of BRMS1 promotes a mesenchymal phenotype through NF- $\mathrm{kB}$-dependent regulation of Twist1. Mol Cell Biol 2015;35:303-17.

13. Lin L, Cai M, Dai Y, et al. Breast cancer metastasis suppressor gene, breast cancer metastasis suppressor 1, may be associated with clinicopathological features of breast cancer. J Cancer Res Ther 2018;14:S368-74.

14. Cui RX, Liu N, He QM, et al. Low BRMS1 expression promotes nasopharyngeal carcinoma metastasis in vitro and in vivo and is associated with poor patient survival. BMC Cancer 2012;12:376.

15. Yang Z, Liu F, Yang ZL. BRMS1 and HPA as Progression,

Cite this article as: Galetta D, Pizzutilo P, Longo V. BRMS1 expression in resected lung adenocarcinoma. Transl Lung Cancer Res 2018;7(Suppl 4):S364-S366. doi: 10.21037/ tlcr.2018.09.20
Clinical Biological Behaviors, and Poor Prognosis-related Biomarkers for Gallbladder Adenocarcinoma. Appl Immunohistochem Mol Morphol 2016;24:275-82.

16. Li J, Cheng Y, Tai D, et al. Prognostic significance of BRMS1 expression in human melanoma and its role in tumor angiogenesis. Oncogene 2011;30:896-906.

17. Smith PW, Liu Y, Siefert SA, et al. Breast cancer metastasis suppressor 1 (BRMS1) suppresses metastasis and correlates with improved patient survival in non-small cell lung cancer. Cancer Lett 2009;276:196-203.

18. Nagji AS, Liu Y, Stelow EB, et al. BRMS1 transcriptional repression correlates with $\mathrm{CpG}$ island methylation and advanced pathological stage in non-small cell lung cancer. J Pathol 2010;221:229-37.

19. Bucciarelli PR, Tan KS, Chudgar NP, et al. BRMS1 Expression in Surgically Resected Lung Adenocarcinoma Predicts Future Metastases and Is Associated with a Poor Prognosis. J Thorac Oncol 2018;13:73-84.

20. Zhang Y, Sun Y, Li Y, et al. ANCCA protein expression is a novel independent poor prognostic marker in surgically resected lung adenocarcinoma. Ann Surg Oncol 2013;20 Suppl 3:S577-82.

21. Yanagawa N, Leduc C, Kohler D, et al. Loss of phosphatase and tensin homolog protein expression is an independent poor prognostic marker in lung adenocarcinoma. J Thorac Oncol 2012;7:1513-21.

22. Dong $X$, Lin D, Low C, et al. Elevated expression of BIRC6 protein in non-small-cell lung cancers is associated with cancer recurrence and chemoresistance. J Thorac Oncol 2013;8:161-70.

23. Xie M, Zhang L, He CS, et al. Prognostic significance of TAZ expression in resected non-small cell lung cancer. J Thorac Oncol 2012;7:799-807.

24. Dhillon T, Mauri FA, Bellezza G, et al. Overexpression of the mammalian target of rapamycin: a novel biomarker for poor survival in resected early stage non-small cell lung cancer. J Thorac Oncol 2010;5:314-9.

25. Zhang J, Qi J, Chen N, et al. High expression of a disintegrin and metalloproteinase-9 predicts a shortened survival time in completely resected stage I non-small cell lung cancer. Oncol Lett 2013;5:1461-6. 